\title{
CONTROL COMMUNICATION COMPLEXITY OF DISTRIBUTED CONTROL SYSTEMS*
}

\author{
WING SHING WONG ${ }^{\dagger}$
}

\begin{abstract}
This paper introduces a measure of communication complexity for a two-agent distributed control system where controls are subject to finite bandwidth communication constraints. The proposed complexity measure is an extension of the idea of communication complexity defined in distributed computing. Applying this classical concept to control problems with finite communication constraints leads to a new perspective and a host of new questions, some of which are investigated in this paper. In particular, one can connect the proposed complexity with the traditional communication complexity via upper bound and lower bound inequalities. Moreover, the proposed complexity is shown to be intricately related to the dynamical characteristics of the underlying system.
\end{abstract}

Key words. DFCB control, communication complexity, distributed control algorithm, control communication complexity

AMS subject classifications. 93A14, 68W15, 91A12

DOI. $10.1137 / 060664720$

1. Introduction. Control under finite bandwidth communication constraints or digital finite communication bandwidth (DFCB) control, a term coined by $\mathrm{Li}$ and Baillieul in [15], has been receiving much attention for the past decade. While germs of related ideas may be found in earlier work on systems with quantized states, such as Delchamps [6], subsequent works, starting with Wong and Brockett [21, 22] and continuing with Tatikonda, Sahai, and Mitter [20], Brockett and Liberzon [3], Nair and Evans [17, 18], Baillieul [1], Elia and Mitter [7], Fagnani and Zampieri [9], Li and Baillieul in [15], Ishii and Francis [12], Tatikonda and Mitter [19], de Persis and Isidori [5], and many others, focus research interests on the interplay among data rates, coding structure, communication protocol, and dynamic behavior of the controlled system, such as containability, asymptotic stability, or observability. Data quantization plays a fundamental role in these investigations, but of equal importance are issues on communication, coding, and complexity. Although all results involving data rates inherently have some implications on system complexity, relatively less attention has been devoted to addressing complexity directly. A notable exception is the paper by Fagnani and Zampieri [10]. Building on results in [8,9], they explicitly introduce various complexity parameters and analyze their implication on system performance.

In theoretical computing, complexity plays a paramount role. There is an influential and thriving program of complexity research based on the concept of communication complexity first introduced by Yao [24]. (For a more detailed description on communication complexity, please refer to [13].) Communication complexity and control under finite communication constraints are ideas coming from two apparently disjoint disciplines. The main contribution of this paper is to marry the two circles of ideas by defining a communication complexity for a controlled dynamical system.

* Received by the editors August 2, 2006; accepted for publication (in revised form) January 19, 2009; published electronically May 20, 2009. This research was partially supported by a grant from the Research Grants Council of the Hong Kong Special Administrative Region under project 417207.

http://www.siam.org/journals/sicon/48-3/66472.html

$\dagger$ Department of Information Engineering, The Chinese University of Hong Kong, Shatin NT, Hong Kong, China (wswong@ie.cuhk.edu.hk). 
To distinguish between the proposed complexity and traditional communication complexity, the former is labeled as the control communication complexity.

Control communication complexity is a new perspective that opens up a host of questions and challenges, of which only a very small portion are discussed in this paper. Nevertheless, preliminary results indicate that there is an intricate relationship among control communication complexity, system dynamics, and system objectives.

To provide motivation for the new concepts introduced here, two application examples will be discussed first. The first concerns mobile sensor networks, which have been serving lately as popular research topics in wireless communication. The second example is a variation of the Leontief input-output economic model. These two examples will be presented in section 2 . In section 3 , the basic model and related concepts from control with finite communication constraints are described. In section 4, a two-agent distributed control system is introduced. The concept of control communication complexity is then defined. Section 5 establishes a connection between the new complexity and traditional communication complexity by means of upper bound and lower bound inequalities. In section 6 , some classes of examples are presented, and their corresponding control communication complexity is analyzed. These examples provide additional insight into the general property of control communication complexity. Concluding remarks are provided in section 7 .

\section{Two motivating examples.}

2.1. Example 1: Mobile sensor network positioning. Consider a sensor network consisting of $k$ remote mobile sensors which are deployed to monitor a geographical area. A network of sensor satellites orbiting in free space is a potential realization of such a network, but for simplicity it is assumed that the sensors are confined to lying on a horizontal plane. The geographical area is divided into two regions, belonging, respectively, to two control agents, Alice and Bob. If one represents a sensor as a point in a configuration space by its position-momentum pair $\left(\mathbf{p}_{i}(t), \mathbf{m}_{i}(t)\right) \in \mathbb{R}^{4}$, then the total system configuration of the network is denoted by the vector $\mathbf{x}=\left(\mathbf{p}_{1}, \mathbf{p}_{2}, \ldots, \mathbf{p}_{k}, \mathbf{m}_{1}, \mathbf{m}_{2}, \ldots, \mathbf{m}_{k}\right)^{T} \in \mathbb{R}^{4 k}$. (In this paper, boldface letters represent vectors or matrices; sets are represented by calligraphic letters.) System dynamics can then be described by an equation of the form

$$
\frac{d \mathbf{x}(t)}{d t}=\mathbf{A} \mathbf{x}(t)+\mathbf{B}_{A} \mathbf{u}_{A}(t)+\mathbf{B}_{B} \mathbf{u}_{B}(t), \quad \mathbf{x}(0)=\mathbf{x}_{0} \in \mathbb{R}^{4 k}
$$

where $\mathbf{u}_{A}$ and $\mathbf{u}_{B}$ represent the respective control of Alice and Bob. The control functions are assumed to be based on digitized observations, and the control values are coded and transmitted over finite bandwidth communication channels.

Depending on the choice of the subregions that require attention, there is an optimal position configuration for the sensors. For illustration, consider a single sensor system in which Alice's region consists of two subregions, $\mathcal{R}_{1}=[-6,-4] \times[-1,1]$ and $\mathcal{R}_{2}=[-2,0] \times[-1,1]$, while Bob's subregions are $\mathcal{S}_{1}=[4,6] \times[-1,1]$ and $\mathcal{S}_{2}=$ $[0,2] \times[-1,1]$. Assume that one subregion from each agent is selected at a time. If sensor accuracy is distance dependent, then for suitably chosen cost functions, one can argue that the optimal position of the sensor is located at the midpoint of the line joining the centers of the subregions to be monitored. These optimal positions can be summarized in the following table for the previously stated example: 


\begin{tabular}{|c|c|c|}
\hline Bob's choice $\backslash$ Alice's choice & $\mathcal{R}_{1}$ & $\mathcal{R}_{2}$ \\
\hline $\mathcal{S}_{1}$ & $(0,0,0,0)^{T}$ & $(2,0,0,0)^{T}$ \\
\hline $\mathcal{S}_{2}$ & $(-2,0,0,0)^{T}$ & $(0,0,0,0)^{T}$ \\
\hline
\end{tabular}

In general, assume that the choices for Alice and Bob are contained in finite sets $\mathcal{A}$ and $\mathcal{B}$, respectively. The optimal configurations can be summarized by a table of size $|\mathcal{A}| \times|\mathcal{B}|$, with entries that are elements in $\mathbb{R}^{4 k}$. The function table and the time-invariant system parameters in (2.1) are known to both agents. It is assumed that Alice chooses an element from $\mathcal{A}$ and Bob chooses an element from $\mathcal{B}$, with the choice unknown to the other agent. The system objective is to define protocols which can guarantee that the system will reach in finite time the state specified according to the agents' choices.

If Alice and Bob do not have direct communication links to each other, a basic question is whether they can jointly steer the network to the optimal state specified by their choices. If the answer to the question is positive, it is natural to investigate how one can quantify the minimum amount of information that needs to be exchanged between the system and the agents in order to achieve this goal.

2.2. Example 2: Control of a Leontief input-output economic model. Consider a dynamic Leontief model $[2,14,16]$ in which an economy is divided into $n$ sectors of industries, with $x_{i}(t)$ representing the gross output rate of the $i$ th sector at time $t$. The gross output rates are adjusted according to the relation

$$
x_{i}(t)=\sum_{j=1}^{n} A_{i j} x_{j}(t)+\sum_{j=1}^{n} B_{i j} \frac{d x_{j}}{d t}(t)+y_{i}(t)
$$

or, in matrix form,

$$
\mathbf{x}(t)=\mathbf{A x}(t)+\mathbf{B} \frac{d \mathbf{x}}{d t}(t)+\mathbf{y}(t) .
$$

Here, the nonnegative input coefficient $A_{i j}$ represents the amount of the $i$ th good needed to produce one unit of the good from the $j$ th sector; $\mathbf{B}$ represents the so-called capital coefficient matrix; $y_{i}(t)$ stands for the exogenous demand of the $i$ th sector at $t$. Unlike in traditional models, it is assumed here that the exogenous demand can be partially controlled by two agents, Alice and Bob, so that

$$
\mathbf{y}(t)=u_{A}(t) \mathbf{y}_{A}+u_{B}(t) \mathbf{y}_{B}+\mathbf{y}_{n}(t),
$$

with $u_{A}(t)$ and $u_{B}(t)$ controlled by Alice and Bob, respectively, and $\mathbf{y}_{n}(t)$ representing the net exogenous output. Suppose that, for a given choice of $\alpha$ for Alice and $\beta$ for Bob, there is a target net exogenous output that they want the system to produce, which can be achieved when $\mathbf{y}_{n}$ reaches the state $\mathbf{y}_{\alpha, \beta}$. The objective for Alice and Bob is to jointly steer the system to such a state at some time instant $T$. If Alice and Bob can communicate their choices directly to each other, this objective may not be difficult to achieve. However, if Alice and Bob do not have a direct communication link (or if they are not allowed to directly communicate due to regulation), can the system objective be accomplished through communication via the system? If the answer to this question is positive, what is the minimum amount of information that needs to be exchanged between the system and the agents? 
Intuitively, the answers to these questions depend on a host of factors, including the structure of the two-agent objective function, the coding functions, as well as observability and controllability conditions of the system. In the following sections, a simple model is proposed so that related questions can be investigated in some detail.

3. Basic dynamical model with communication constraints. The source of this work can be traced to control systems under finite bandwidth communication constraints and Yao's concept of communication complexity. To acquaint readers with the former circle of ideas, consider the following DFCB control model:

$$
\left\{\begin{array}{l}
\mathbf{x}_{t}=\mathbf{D x}_{t-1}+\mathbf{E} \mathbf{u}_{t-1}\left(\zeta_{t-1}\right), \quad \mathbf{x}_{0} \in \mathcal{X}_{0} \subseteq \mathbb{R}^{n} \\
\psi_{t}=Q_{t}\left(\mathbf{C x}_{t}\right) \\
\zeta_{t}=K_{t}\left(\psi_{t}, \alpha\right)
\end{array}\right.
$$

The control coefficient matrix $\mathbf{E}$ is of dimension $n \times m$, and the observation matrix $\mathbf{C}$ is of dimension $\ell \times n$. The function $Q_{t}: \mathbb{R}^{\ell} \rightarrow \mathcal{L}_{t}$ is a time-dependent observation quantization/encoding function taking value in a finite set $\mathcal{L}_{t} ; K_{t}: \mathcal{L}_{t} \times \mathcal{A} \rightarrow \mathcal{M}_{t}$ denotes a feedback control law that depends on a finite parameter set $\mathcal{A}$, which determines the operating point of the system, and maps an observation code-word from $\mathcal{L}_{t}$ into a control code-word taking values in a finite set $\mathcal{M}_{t}$. The function $\mathbf{u}(\cdot): \mathcal{M}_{t} \rightarrow \mathbb{R}^{m}$ maps a control code-word to a control vector. Lastly, $\mathcal{X}_{0}$ represents a known set of initial states.

Equation (3.1) can be interpreted as defining a control system with a distributed feedback controller. At time $t$, the observer generates the observation output $\mathbf{C x}_{t-1}$ and quantizes it by means of the function $Q_{t}$ into $\lambda_{t}$ bits, where

$$
\lambda_{t}=\left\lceil\log _{2}\left|\mathcal{L}_{t}\right|\right\rceil
$$

These observation bits are then sent to the decision-maker. Control decision is computed by the decision-maker via $K_{t}$ and coded into $\mu_{t}$ control bits, where

$$
\mu_{t}=\left\lceil\log _{2}\left|\mathcal{M}_{t}\right|\right\rceil
$$

Thus, for each observation-control cycle, $\lambda_{t}+\mu_{t}$ bits of information will be exchanged between the decision-maker and the physical plant. Figure 1 shows a schematic of the distributed control system.

The second inspiration of this paper comes from communication complexity, which is, roughly speaking, a measure of the communication required for two agents to jointly compute a function by means of a distributed protocol. Several closely related definitions of communication complexity have been proposed subsequent to Yao's original definition. The version presented in [13] is adopted here. Let $\mathcal{X}, \mathcal{Y}$, and let $\mathcal{Z}$ be finite sets and $f$ be a function from $\mathcal{X} \times \mathcal{Y}$ to $\mathcal{Z}$. In [13], a protocol is formally defined as a binary tree where each of its internal nodes is identified with a binary valued function affiliated with either Alice or Bob and each leaf node is affiliated with an element in $\mathcal{Z}$. To carry out the computation when Alice chooses $\alpha$ from $\mathcal{X}$ and Bob chooses $\beta$ from $\mathcal{Y}$, one starts from the root node and computes at each internal node the affiliated function using either $\alpha$ or $\beta$ as an argument, depending on whether the node function is affiliated with Alice or Bob. One moves to the left child if the function evaluates to zero and moves to the right child if it evaluates to one. Output of the protocol is taken to be the value affiliated with the leaf node reached in the end. The cost of a protocol is the height of the tree. The communication complexity of $f$, 


\section{Decision-Maker}

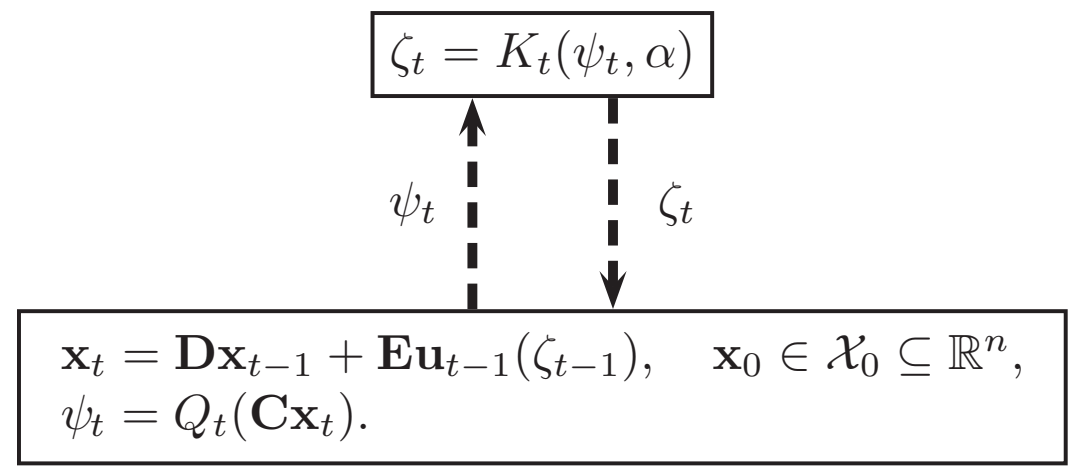

\section{Physical Plant}

FIG. 1. A DFCB control model.

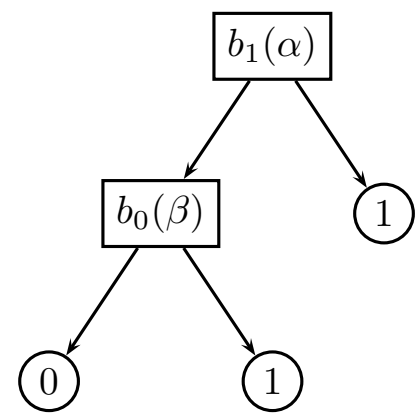

FIG. 2. Example of a protocol computing the function $f$.

denoted by $D(f)$, is then defined to be the minimum cost among all protocols that compute $f$ correctly. In this definition of communication complexity, both agents are required to know the computed value when the protocol halts.

As a simple example, consider the computation of the following binary function, $f:\{0,1\} \times\{0,1\} \rightarrow\{0,1\}:$

\begin{tabular}{|c|c|c|}
\hline$\beta \backslash \alpha$ & 0 & 1 \\
\hline 0 & 1 & 1 \\
\hline 1 & 0 & 1 \\
\hline
\end{tabular}

For $i=\{0,1\}$ let $b_{i}(\gamma)$ be a binary function that is equal to 1 if $\gamma=i$ and 0 otherwise. Then, the binary tree in Figure 2 describes a correct protocol to compute $f$ that starts with Alice.

To see why this protocol computes correctly, interpret each node in the graph as a step of having one communication bit exchanged between the agents. Thus, the first node represents the step that a bit is sent from Alice to Bob carrying the outcome of the function, $b_{1}(\alpha)$. If $b_{1}(\alpha)$ is equal to 1 , then both agents can conclude that $f(\alpha, \beta)=1$. Otherwise, one more bit of information needs to be exchanged, this time 
from Bob to Alice. In this example, the depth of the tree is 2, and it can be shown that for this function $D(f)$ is indeed 2. For the original definition of communication complexity by Yao, denoted here by $C(f)$, the protocol stops when either Alice or Bob can determine the value of $f$. For the protocol defined in Figure 2, this means that the protocol can stop after one bit has been exchanged. So, in this case,

$$
D(f)=C(f)+1 .
$$

In the next section, a two-agent distributed control system is introduced, which is based on a DFCB control model with a structure akin to the system defined in (3.1). The concept of communication complexity will then be defined.

Subsequent discussions of the coded observer require the concept of quantization by means of a lattice [4]. A lattice $\Lambda$ generated by an $n \times n$ full rank matrix, $\mathbf{Z}=$ $\left(\mathbf{z}_{1}, \ldots, \mathbf{z}_{n}\right)$, consists of points in $\mathbb{R}^{n}$ of the form

$$
\left\{\sum_{i=1}^{n} m_{i} \mathbf{z}_{i}: m_{i} \in \mathbb{Z}, i=1, \ldots, n\right\} .
$$

For any $\left(m_{1}, \ldots, m_{n}\right) \in \mathbb{Z}^{n}$, a cell $\mathcal{X}$ centered at $\sum_{i=1}^{n} m_{i} \mathbf{z}_{i}$ is a subset of $\mathbb{R}^{n}$ :

$$
\left\{\sum_{i=1}^{n}\left(m_{i}+\epsilon_{i}\right) \mathbf{z}_{i}:-1 / 2 \leq \epsilon_{i}<1 / 2, i=1, \ldots, n\right\} .
$$

When a quantization function is defined via a lattice, all elements in a cell of the lattice are represented by the cell center. Given a full rank $n \times n$ matrix $\mathbf{H}$, one can define a new lattice $\mathbf{H} \Lambda$ by

$$
\mathbf{H} \Lambda=\left\{\sum_{i=1}^{n} m_{i} \mathbf{H z}_{i}: m_{i} \in \mathbb{Z}, i=1, \ldots, n\right\} .
$$

If $\mathcal{X}$ is a cell of $\Lambda$ centered at $\mathbf{x}$, then $\mathbf{H} \mathcal{X}$ is a cell of $\mathbf{H} \Lambda$ centered at $\mathbf{H x}$.

4. A distributed control system and its communication complexity. Consider a two-agent distributed control system $\boldsymbol{\Sigma}$, which is defined by the following system of equations. For $t \geq 0$,

$$
\left\{\begin{array}{c}
\mathbf{x}_{t+1}=\mathbf{D} \mathbf{x}_{t}+\mathbf{E}_{A} \mathbf{u}_{t}^{(A)}\left(\zeta_{t}^{(A)}, \ldots, \zeta_{0}^{(A)}, \psi_{t}^{(A)}, \ldots, \psi_{0}^{(A)}\right) \\
\quad+\mathbf{E}_{B} \mathbf{u}_{t}^{(B)}\left(\zeta_{t}^{(B)}, \ldots, \zeta_{0}^{(B)}, \psi_{t}^{(B)}, \ldots, \psi_{0}^{(B)}\right) \\
\mathbf{x}_{0} \in \mathcal{X}_{0} \subseteq \mathbb{R}^{n} \\
\psi_{t}^{(A)}=Q_{t}^{(A)}\left(\mathbf{C}_{A} \mathbf{x}_{t}, \ldots, \mathbf{C}_{A} \mathbf{x}_{0}\right), \quad \psi_{t}^{(B)}=Q_{t}^{(B)}\left(\mathbf{C}_{B} \mathbf{x}_{t}, \ldots, \mathbf{C}_{B} \mathbf{x}_{0}\right) \\
\zeta_{t}^{(A)}=K_{t}^{(A)}\left(\psi_{t}^{(A)}, \ldots, \psi_{0}^{(A)}, \alpha\right), \quad \zeta_{t}^{(B)}=K_{t}^{(B)}\left(\psi_{t}^{(B)}, \ldots, \psi_{0}^{(B)}, \beta\right) .
\end{array}\right.
$$

Here, $\mathbf{C}_{A}$ and $\mathbf{C}_{B}$ are the observation matrices of dimension $\ell_{A} \times n$ and $\ell_{B} \times n$, respectively; $\mathbf{E}_{A}$ and $\mathbf{E}_{B}$ are control matrices of dimension $n \times m_{A}$ and $n \times m_{B}$, respectively. $Q_{t}^{(A)}$ and $Q_{t}^{(B)}$ are quantization/coding functions mapping the observations to finite sets, with their domains and ranges defined by

$$
Q_{t}^{(A)}: \underbrace{\mathbb{R}^{\ell_{A}} \times \cdots \times \mathbb{R}^{\ell_{A}}}_{t+1} \rightarrow \mathcal{L}_{t}^{(A)}, \quad Q_{t}^{(B)}: \underbrace{\mathbb{R}^{\ell_{B}} \times \cdots \times \mathbb{R}^{\ell_{B}}}_{t+1} \rightarrow \mathcal{L}_{t}^{(B)},
$$




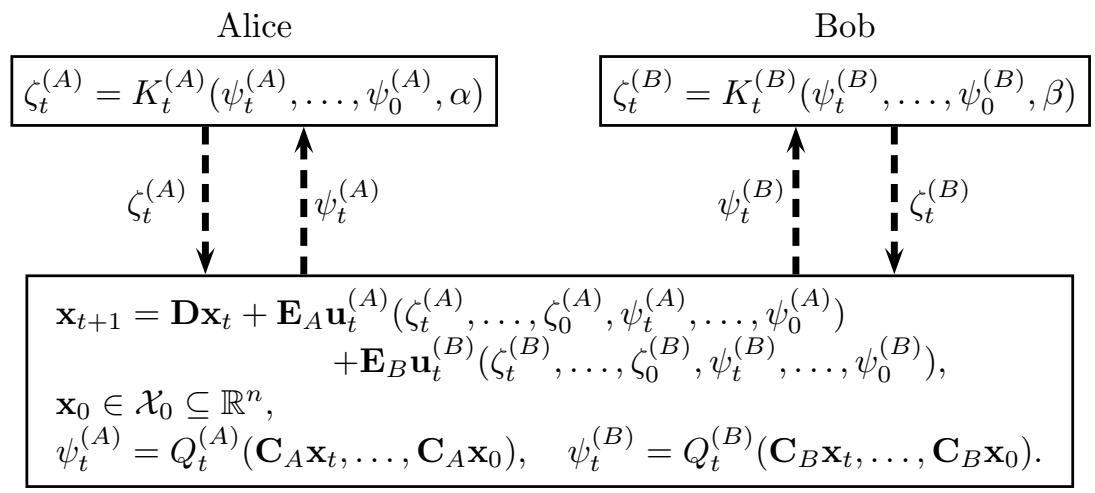

Dynamical System

FiG. 3. A distributed control system.

where $\mathcal{L}_{t}^{(A)}$ and $\mathcal{L}_{t}^{(B)}$ are finite sets. Without loss of generality, the mappings $Q_{t}^{(A)}$ and $Q_{t}^{(B)}$ are assumed to be surjective. The coded observations $\psi_{t}^{(A)}$ and $\psi_{t}^{(B)}$ are sent to Alice and Bob, respectively, over digital communication channels. If the observation coding function is a constant, then no communication bit needs to be exchanged. Alice and Bob determine the appropriate control code-words by means of the functions $K_{t}^{(A)}$ and $K_{t}^{(B)}$, respectively. The domains and ranges of these functions are defined as

$$
\begin{aligned}
K_{t}^{(A)} & : \mathcal{L}_{t}^{(A)} \times \cdots \times \mathcal{L}_{0}^{(A)} \times \mathcal{A} \rightarrow \mathcal{M}_{t}^{(A)}, \\
K_{t}^{(B)} & : \mathcal{L}_{t}^{(B)} \times \cdots \times \mathcal{L}_{0}^{(B)} \times \mathcal{B} \rightarrow \mathcal{M}_{t}^{(B)} .
\end{aligned}
$$

Here, $\mathcal{A}$ and $\mathcal{B}$ are finite sets containing the choices of Alice and Bob, respectively. Without loss of generality, one can assume that $K_{t}^{(A)}$ and $K_{t}^{(B)}$ are surjective.

The dynamical system keeps a bank of control mappings for each agent. It can select which mapping to use based on the observation code-words that have been sent to that agent up to the moment. (In other words, the system remembers what observation code-words have been sent to a particular agent.) Alice activates her control by means of the control code-word sequence $\left\{\zeta_{0}^{(A)}, \ldots, \zeta_{t}^{(A)}\right\}$, and Bob activates his control by means of $\left\{\zeta_{0}^{(B)}, \ldots, \zeta_{t}^{(B)}\right\}$. Thus, the domain and range of the control functions can be formulated as follows:

$$
\begin{aligned}
& \mathbf{u}_{t}^{(A)}(\cdot): \mathcal{M}_{t}^{(A)} \times \cdots \times \mathcal{M}_{0}^{(A)} \times \mathcal{L}_{t}^{(A)} \times \cdots \times \mathcal{L}_{0}^{(A)} \rightarrow \mathbb{R}^{m_{A}}, \\
& \mathbf{u}_{t}^{(B)}(\cdot): \mathcal{M}_{t}^{(B)} \times \cdots \times \mathcal{M}_{0}^{(B)} \times \mathcal{L}_{t}^{(B)} \times \cdots \times \mathcal{L}_{0}^{(B)} \rightarrow \mathbb{R}^{m_{B}} .
\end{aligned}
$$

The functions $\mathbf{u}_{t}^{(A)}$ and $\mathbf{u}_{t}^{(B)}$ are of infinite precision and are preloaded before the dynamical system is activated. (It is possible to assume a simpler structure for the control functions so that they depend only on the control code-words sent by the agents. Since the control code-words could include information about the coded observations, this simpler function structure can achieve controlling effects that are identical to those of the current approach but at a higher communication cost.)

Definition 1. A distributed control protocol for the system $\boldsymbol{\Sigma}$ is defined by the functions $\left\{Q_{t}^{(A)}\right\}_{t=0}^{\infty},\left\{Q_{t}^{(B)}\right\}_{t=0}^{\infty},\left\{K_{t}^{(A)}\right\}_{t=0}^{\infty},\left\{K_{t}^{(B)}\right\}_{t=0}^{\infty},\left\{\mathbf{u}_{t}^{(A)}\right\}_{t=0}^{\infty}$, and $\left\{\mathbf{u}_{t}^{(B)}\right\}_{t=0}^{\infty}$.

A schematic of the control system is shown in Figure 3. 
4.1. System parameters and protocol operation. It would be informative to describe for the functions in a control protocol the system parameters on which they are allowed to depend. These parameters may include

S1. the sets $\mathcal{A}$ and $\mathcal{B}$ and the target function $f$,

S2. the matrices $\mathbf{D}, \mathbf{E}_{A}, \mathbf{E}_{B}, \mathbf{C}_{A}$, and $\mathbf{C}_{B}$,

S3. the initial state set $\mathcal{X}_{0}$.

Before the controls are activated, the functions $\left\{K_{t}^{(A)}\right\}_{t=0}^{\infty}$ are distributed to Alice, $\left\{K_{t}^{(B)}\right\}_{t=0}^{\infty}$ are distributed to Bob, and the functions $\left\{Q_{t}^{(A)}\right\}_{t=0}^{\infty},\left\{Q_{t}^{(B)}\right\}_{t=0}^{\infty},\left\{\mathbf{u}_{t}^{(A)}\right\}_{t=0}^{\infty}$, and $\left\{\mathbf{u}_{t}^{(B)}\right\}_{t=0}^{\infty}$ are implemented at the dynamical system. The agents and the dynamical system are synchronized to start at time 0 and iteratively compute these functions until the stopping condition is satisfied.

4.2. Information structure and agent knowledge. In order to explain the stopping rule, it is helpful to provide a precise meaning of the statement that an agent has knowledge of a function $f$.

It is common to define information as the observation available to the decisionmaker (see, for example, [11]). Represent the information available to Alice and Bob at time $t$ by $\mathcal{I}_{A}(t)$ and $\mathcal{I}_{B}(t)$, respectively. Then,

$$
\begin{array}{ll}
\mathcal{I}_{A}(0)=\left(\psi_{0}^{(A)}, \alpha\right), & \mathcal{I}_{A}(1)=\left(\psi_{1}^{(A)}, \psi_{0}^{(A)}, \alpha\right), \ldots, \\
\mathcal{I}_{B}(0)=\left(\psi_{0}^{(B)}, \beta\right), & \mathcal{I}_{B}(1)=\left(\psi_{1}^{(B)}, \psi_{0}^{(B)}, \beta\right), \ldots
\end{array}
$$

(Since part or all of the system parameters S1, S2, and S3 could be included in the definition of the functions $\left\{K_{t}^{(A)}\right\}_{t=0}^{\infty}$ and $\left\{K_{t}^{(B)}\right\}_{t=0}^{\infty}$, these parameters can be regarded as a priori information known to the agents.)

Although a parameter may not be directly observable, an agent may be able to deduce its value with certainty from prior knowledge or indirect observations. For example, although Bob does not have direct observation on $\alpha$, he can determine its value from his knowledge of $\mathcal{A}$ if it is a singleton set. To make this concept precise, the following definition is introduced. Let $f_{1}, \ldots, f_{p}$ and $f_{0}$ be real functions defined on a common set of real variables $\left(z_{1}, \ldots, z_{K}\right)$.

Definition 2. An agent is said to have knowledge of $f_{0}$ based on the information $\left(r_{1}, \ldots, r_{p}\right)$, which is an instantiation of the functions $\left(f_{1}, \ldots, f_{p}\right)$, if the set

$$
\left\{f_{0}\left(z_{1}, \ldots, z_{K}\right) \in \mathbb{R}: f_{1}\left(z_{1}, \ldots, z_{K}\right)=r_{1}, \ldots, f_{p}\left(z_{1}, \ldots, z_{K}\right)=r_{p}\right\}
$$

is a singleton.

In a traditional communication complexity model, a protocol is specified by a binary tree and the functions affiliated with its internal nodes. These functions take either $\alpha$ or $\beta$ as argument. The bits that are exchanged between the agents represent the values of these functions. A protocol correctly computes a function $f$ if and only if the agents have knowledge of $f$ for any instantiation of the node functions.

4.3. System objective and stopping rule. The system objective is to design a distributed control protocol that enables the agents to achieve a common goal that is specified by a target function. There are two types of target functions considered in this paper.

Type A. This type of target function is used when and only when the initial set is finite. $f$ is a function from $\mathcal{A} \times \mathcal{B}$ to the state space $\mathbb{R}^{n}$ and represents the state to which Alice and Bob aim to control the system to reach in finite time. 
Type B. This type of target function is used when and only when the initial set is infinite. $f$ is a function from $\mathcal{A} \times \mathcal{B}$ to cells defined by a lattice $\Lambda$ on $\mathbb{R}^{n}$. The function represents the cell to which Alice and Bob aim to control the system to enter in finite time.

Denote $f(\mathcal{A} \times \mathcal{B})$ by $\mathcal{F}_{R}$. It follows that the cardinality of $\mathcal{F}_{R}$ satisfies

$$
\left|\mathcal{F}_{R}\right| \leq|\mathcal{A}| \cdot|\mathcal{B}|
$$

Since $\mathcal{F}_{R}$ is a finite set, the computation complexity of $f$ in the traditional sense is well defined.

All functions in a distributed protocol sequence, and hence all information available to the agents, can ultimately be regarded as functions that take $\alpha, \beta$, and $\mathbf{x}_{0}$ as arguments. To ensure that the agents have knowledge of $f$ is one of the key objectives of the distributed control system considered here. At the same time, it is important to guarantee that the system enter the target state at some finite time. Therefore, formally stated, the objectives of a distributed control protocol are to ensure that, for any choice $\alpha \in \mathcal{A}$ of Alice, any choice $\beta \in \mathcal{B}$ of Bob, and any initial state $\mathbf{x}_{0} \in \mathcal{X}_{0}$, the following hold:

1. There is a finite time $\sigma$ such that, based on $\mathcal{I}_{A}(\sigma)$, Alice has knowledge of $f$, and based on $\mathcal{I}_{B}(\sigma)$, Bob has knowledge of $f$.

2. There is a finite time $\tau$ such that

$$
\begin{aligned}
& \mathbf{x}_{\tau}\left(\mathbf{x}_{0}\right)=f(\alpha, \beta) \text { for a Type A target function, } \\
& \mathbf{x}_{\tau}\left(\mathbf{x}_{0}\right) \in f(\alpha, \beta) \quad \text { for a Type B target function, }
\end{aligned}
$$

where $\mathbf{x}_{t}\left(\mathbf{x}_{0}\right)$ is the trajectory $\mathbf{x}_{t}$ of (4.1) starting from $\mathbf{x}_{0}$.

3. There is a finite time $\rho$ such that, based on $\mathcal{I}_{A}(\rho)$, Alice has knowledge of $\tau$, and based on $\mathcal{I}_{B}(\rho)$, Bob has knowledge of $\tau$.

The system is considered terminated after time $\max (\sigma, \tau, \rho)$. In the definition of the system objective, there is no condition on how $\sigma, \tau$, and $\rho$ should relate to each other. The intention is to allow flexibility in a generic model. It is possible, for example, to refine the model by requiring that $\tau \geq \sigma$.

A distributed control protocol that accomplishes these objectives for any $\alpha, \beta$, and $\mathbf{x}_{0}$ is known as a correct protocol.

4.4. Quantifying the information exchange. To measure the amount of data that needs to be exchanged at time $t$, define

$$
\begin{array}{ll}
\lambda_{t}^{(A)}=\left\lceil\log _{2}\left|\mathcal{L}_{t}^{(A)}\right|\right\rceil, & \mu_{t}^{(A)}=\left\lceil\log _{2}\left|\mathcal{M}_{t}^{(A)}\right|\right\rceil, \\
\lambda_{t}^{(B)}=\left\lceil\log _{2}\left|\mathcal{L}_{t}^{(B)}\right|\right\rceil, & \mu_{t}^{(B)}=\left\lceil\log _{2}\left|\mathcal{M}_{t}^{(B)}\right|\right\rceil .
\end{array}
$$

If no information is communicated, the convention is to set the corresponding function to a constant. Clearly there is no loss of generality in assuming that for $t \geq \max (\sigma, \tau, \rho)$ no communication bits are exchanged and that

$$
\lambda_{t}^{(A)}=\mu_{t}^{(A)}=\lambda_{t}^{(B)}=\mu_{t}^{(B)}=0 .
$$

4.5. Rundown of a control cycle. To further illustrate the basic concepts, it is helpful to describe the rundown of a control cycle. To start the first control cycle, the system quantizes the observation $\mathbf{C}_{A} \mathbf{x}_{0}$ into $\psi_{0}^{(A)}$ and the observation $\mathbf{C}_{B} \mathbf{x}_{0}$ 
into $\psi_{0}^{(B)}$. These coded observations are sent to Alice and Bob, respectively. Alice then computes the control code-word $\zeta_{0}^{(A)}$ by means of $K_{0}^{(A)}$ using $\psi_{0}^{(A)}$ and $\alpha$ as arguments. Similarly, $\zeta_{0}^{(B)}$ is computed via $K_{0}^{(B)}$ by Bob using $\psi_{0}^{(B)}$ and $\beta$. The control code-words are then forwarded to the physical system and are mapped into the corresponding controls, $\mathbf{u}_{0}^{(A)}$ and $\mathbf{u}_{0}^{(B)}$, referred by $\left(\zeta_{0}^{(A)}, \psi_{0}^{(A)}\right)$ and $\left(\zeta_{0}^{(B)}, \psi_{0}^{(B)}\right)$, respectively. Equation (4.1) is then updated with these controls. This completes the first control cycle. Subsequent control cycles can be described similarly.

4.6. Control communication complexity. Given a distributed control protocol $\Omega$ for a distributed control system $(\boldsymbol{\Sigma}, f)$, and given an initial choice of $\alpha, \beta$, and $\mathbf{x}_{0}$, the number of bits exchanged between the agents and the system is summed up by the formula

$$
c\left(\Omega, \alpha, \beta, \mathbf{x}_{0}\right)=\sum_{t=0}^{\infty}\left(\lambda_{t}^{(A)}+\lambda_{t}^{(B)}+\mu_{t}^{(A)}+\mu_{t}^{(B)}\right) .
$$

For a fixed protocol $\Omega$, this sum is a deterministic function defined on $\mathcal{A} \times \mathcal{B} \times \mathcal{X}_{0}$ and has a supremum which is denoted by $C(\Omega)$. That is,

$$
C(\Omega)=\sup _{\left(\alpha, \beta, \mathbf{x}_{0}\right)} c\left(\Omega, \alpha, \beta, \mathbf{x}_{0}\right) .
$$

DeFINITION 3. The control communication complexity of a distributed control system $(\boldsymbol{\Sigma}, f)$ is defined to be the minimum of $C(\Omega)$ as $\Omega$ varies over the set of correct distributed control protocols. That is,

$$
Y(\boldsymbol{\Sigma}, f)=\min _{\Omega} C(\Omega)
$$

$Y(\boldsymbol{\Sigma}, f)$ is closely related to the traditional concept of communication complexity of $f$.

5. Basic bounds for control communication complexity. In this section some basic bounds of the newly defined complexity are introduced.

Theorem 1. For any distributed control system $(\boldsymbol{\Sigma}, f)$,

$$
2 D(f) \leq Y(\boldsymbol{\Sigma}, f) .
$$

Proof. Let $\Omega$ be a correct distributed control protocol that achieves the complexity $Y(\boldsymbol{\Sigma}, f)$. For any $\mathbf{x}_{0}$ in the initial state set and for any choices $\alpha$ and $\beta$, the following holds:

$$
c\left(\Omega, \alpha, \beta, \mathbf{x}_{0}\right) \leq Y(\boldsymbol{\Sigma}, f) .
$$

Now consider a modified distributed control system in which the dynamical system always starts from the state $\mathbf{x}_{0}$ which is known to both Alice and Bob. Regard the dynamical system and Alice as forming a single entity and label it as Super Alice. Consider the traditional communication complexity of computing $f$ between Bob and Super Alice. Viewed in this manner, any distributed control algorithm for the original problem can be reinterpreted to provide a solution to the traditional problem. For 
example, one can view the system

$$
\left\{\begin{array}{l}
\mathbf{x}_{t+1}=\mathbf{D} \mathbf{x}_{t}+\mathbf{E}_{A} \mathbf{u}_{t}^{(A)}\left(\zeta_{t}^{(A)}, \ldots, \zeta_{0}^{(A)}, \psi_{t}^{(A)}, \ldots, \psi_{0}^{(A)}\right) \\
\quad+\mathbf{E}_{B} \mathbf{u}_{t}^{(B)}\left(\zeta_{t}^{(B)}, \ldots, \zeta_{0}^{(B)}, \psi_{t}^{(B)}, \ldots, \psi_{0}^{(B)}\right) \\
\quad \mathbf{x}_{0} \text { given, } \\
\psi_{t}^{(A)}=Q_{t}^{(A)}\left(\mathbf{C}_{A} \mathbf{x}_{t}, \ldots, \mathbf{C}_{A} \mathbf{x}_{0}\right), \quad \psi_{t}^{(B)}=Q_{t}^{(B)}\left(\mathbf{C}_{B} \mathbf{x}_{t}, \ldots, \mathbf{C}_{B} \mathbf{x}_{0}\right), \\
\zeta_{t}^{(A)}=K_{t}^{(A)}\left(\psi_{t}^{(A)}, \ldots, \psi_{0}^{(A)}, \alpha\right)
\end{array}\right.
$$

as a computing device for Alice that takes the sequence $\left\{\zeta_{t}^{(B)}\right\}$ as input and generates the sequence $\left\{\psi_{t}^{(B)}\right\}$ as output. This offers a solution to the traditional distributed computation problem of $f$ between Bob and Super Alice. Hence,

$$
\sum_{t=0}^{\infty}\left(\lambda_{t}^{(B)}+\mu_{t}^{(B)}\right) \geq D(f) .
$$

A similar argument leads to a corresponding inequality for $\sum_{t=0}^{\infty}\left(\lambda_{t}^{(A)}+\mu_{t}^{(A)}\right)$. Hence,

$$
2 D(f) \leq c\left(\Omega, \alpha, \beta, \mathbf{x}_{0}\right) \leq Y(\boldsymbol{\Sigma}, f) .
$$

Definition 4. Given a bounded subset of $\mathbb{R}^{n}, \mathcal{X}$, and a lattice $\Lambda$ of $\mathbb{R}^{n}$, the order of $\mathcal{X}$ over $\Lambda$, denoted by $O(\mathcal{X}, \Lambda)$, is equal to the number of distinct cells of $\Lambda$ that have nonempty intersection with $\mathcal{X}$.

For example, on a two-dimensional plane, the open unit disc centered at the origin has an order of 9 over the lattice generated by $\{(0,1),(1,0)\}$.

Definition 5. The triple $\left(\mathbf{D}, \mathbf{E}_{A}, \mathbf{E}_{B}\right)$ is jointly controllable if the matrix

$$
\left(\mathbf{E}_{A}, \mathbf{E}_{B}, \mathbf{D} \mathbf{E}_{A}, \mathbf{D} \mathbf{E}_{B}, \mathbf{D}^{2} \mathbf{E}_{A}, \mathbf{D}^{2} \mathbf{E}_{B}, \ldots, \mathbf{D}^{n-1} \mathbf{E}_{A}, \mathbf{D}^{n-1} \mathbf{E}_{B}\right)
$$

has rank equal to $n$.

For a jointly controllable triple, let $n_{A}$ be the rank of the matrix $\left(\mathbf{E}_{A}, \mathbf{D E}_{A}, \ldots\right.$, $\left.\mathbf{D}^{n-1} \mathbf{E}_{A}\right)$; let $\mathcal{B}_{A}=\left\{\mathbf{e}_{1}, \ldots, \mathbf{e}_{n_{A}}\right\}$ be a set of independent columns chosen from it. Let $\mathcal{B}_{B}=\left\{\mathbf{f}_{1}, \ldots, \mathbf{f}_{n-n_{A}}\right\}$ be a set of independent columns chosen from the matrix $\left(\mathbf{E}_{B}, \mathbf{D} \mathbf{E}_{B}, \ldots, \mathbf{D}^{n-1} \mathbf{E}_{B}\right)$ that are independent of $\left\{\mathbf{e}_{1}, \ldots, \mathbf{e}_{n_{A}}\right\}$. Such a set exists since the matrix (5.6) is of rank $n$. The union $\mathcal{B}_{A} \cup \mathcal{B}_{B}$ forms a basis of $\mathbb{R}^{n}$, not necessarily unique or orthogonal.

Given a jointly controllable triple $\left(\mathbf{D}, \mathbf{E}_{A}, \mathbf{E}_{B}\right)$ and a base $\mathcal{B}_{A} \cup \mathcal{B}_{B}$, any element $\mathbf{x}$ in $\mathbb{R}^{n}$ has a unique representation

$$
\mathbf{x}=\mathbf{x}_{A}+\mathbf{x}_{B}
$$

so that $\mathbf{x}_{A}$ is contained in the subspace spanned by $\mathcal{B}_{A}$ and $\mathbf{x}_{B}$ is contained in the subspace spanned by $\mathcal{B}_{B}$. Denote the mappings taking $\mathbf{x}$ to $\mathbf{x}_{A}$ and $\mathbf{x}_{B}$ by $P_{A}$ and $P_{B}$, respectively.

TheOrem 2. Suppose that the triple $\left(\mathbf{D}, \mathbf{E}_{A}, \mathbf{E}_{B}\right)$ is jointly controllable with a base given by $\mathcal{B}_{A} \cup \mathcal{B}_{B}$, the ordered pairs $\left(\mathbf{D}, \mathbf{C}_{A}\right)$ and $\left(\mathbf{D}, \mathbf{C}_{B}\right)$ are both observable, $\mathbf{C}_{B} \mathbf{E}_{A} \neq 0$, and $\mathbf{C}_{A} \mathbf{E}_{B} \neq 0$. Then for any distributed control system $(\boldsymbol{\Sigma}, f)$ with a finite initial set and a Type A target function, the following inequality holds:

$$
Y(\boldsymbol{\Sigma}, f) \leq 4 D(f)+4\left\lceil\log _{2}\left|\mathcal{X}_{0}\right|\right\rceil+2\left\lceil\log _{2}\left|\mathcal{F}_{R}\right|\right\rceil .
$$


Proof. To prove the bound, consider a protocol $\Pi$ which achieves the communication complexity for the affiliated traditional computation problem. Based on it, one can construct a distributed control protocol which consists of three phases. In the first phase, the initial state of the system is identified and communicated to the agents. In the second phase, Alice and Bob communicate to each other by replicating the bit sequences defined in $\Pi$. In the third and final phase, Alice and Bob jointly steer the system to reach the target state at a fixed time, $2 n+D(f)-1$.

The first phase starts from time 0 and ends when the quantized code-words of the observed state $\mathbf{x}_{n-1}$ are communicated to the agents. In this phase all controls are set to zero. It follows that $\mathbf{x}_{t}=\mathbf{D}^{t} \mathbf{x}_{0}$ for $0 \leq t \leq n-1$. Hence

$$
\begin{aligned}
\left(\begin{array}{c}
\mathbf{C}_{A} \mathbf{x}_{0} \\
\mathbf{C}_{A} \mathbf{x}_{1} \\
\vdots \\
\mathbf{C}_{A} \mathbf{x}_{n-1}
\end{array}\right)=\left(\begin{array}{c}
\mathbf{C}_{A} \mathbf{x}_{0} \\
\mathbf{C}_{A} \mathbf{D} \mathbf{x}_{0} \\
\vdots \\
\mathbf{C}_{A} \mathbf{D}^{n-1} \mathbf{x}_{0}
\end{array}\right) \equiv \mathbf{H}_{A} \mathbf{x}_{0} \\
\left(\begin{array}{c}
\mathbf{C}_{B} \mathbf{x}_{0} \\
\mathbf{C}_{B} \mathbf{x}_{1} \\
\vdots \\
\mathbf{C}_{B} \mathbf{x}_{0} \\
\mathbf{C}_{B} \mathbf{D} \mathbf{x}_{0} \\
\vdots \\
\mathbf{C}_{B} \mathbf{D}^{n-1} \mathbf{x}_{0}
\end{array}\right) \equiv \mathbf{H}_{B} \mathbf{x}_{0} .
\end{aligned}
$$

By the observable assumption, there exist $n$ independent rows in $\mathbf{H}_{A}$ and $n$ independent rows in $\mathbf{H}_{B}$ so that the resulting submatrices are invertible. Hence, one can determine $\mathbf{x}_{0}$ from the observation sequence $\left\{\mathbf{C}_{A} \mathbf{x}_{0}, \mathbf{C}_{A} \mathbf{x}_{1}, \ldots, \mathbf{C}_{A} \mathbf{x}_{n-1}\right\}$. Since the initial state set is finite, one can define a quantization function $Q_{n-1}^{(A)}$ taking this observation sequence as arguments to uniquely identify $\mathbf{x}_{0}$ by a finite code-word. Similarly, one can define a function $Q_{n-1}^{(B)}$ taking $\left\{\mathbf{C}_{B} \mathbf{x}_{0}, \mathbf{C}_{B} \mathbf{x}_{1}, \ldots, \mathbf{C}_{B} \mathbf{x}_{n-1}\right\}$ as arguments to uniquely identify $\mathbf{x}_{0}$ by a finite code-word. The number of code-words needed to identify the different initial states is equal to the cardinality of $\mathcal{X}_{0}$. Hence, one can construct these quantization functions so that

$$
\left|\mathcal{L}_{n-1}^{(A)}\right|=\left|\mathcal{L}_{n-1}^{(B)}\right|=\left|\mathcal{X}_{0}\right| .
$$

At the end of phase 1, the code-word representing $\mathbf{x}_{0}$ is sent to Alice and Bob. The total number of bits exchanged in this phase is

$$
2\left\lceil\log _{2}\left|\mathcal{X}_{0}\right|\right\rceil \text {. }
$$

The second phase starts with the computation of the control code-words $\zeta_{n-1}^{(A)}$ and $\zeta_{n-1}^{(B)}$ and ends at the delivery of the code-words $\psi_{n+D(f)-1}^{(A)}$ and $\psi_{n+D(f)-1}^{(B)}$ to the agents. During this phase, the protocol $\Pi$ is transcribed to the distributed control system.

To explain how the transcription is done, assume without loss of generality that the first bit is sent by Alice in $\Pi$, then set $\zeta_{n-1}^{(A)}$ to 1 or -1 depending on whether the value of the bit sent is 1 or 0 . Let $\mathbf{v}_{A} \in \mathbb{R}^{m_{A}}$ be a vector satisfying $\mathbf{C}_{B} \mathbf{E}_{A} \mathbf{v}_{A} \neq 0$. (The existence of such a vector is guaranteed by the assumption $\mathbf{C}_{B} \mathbf{E}_{A} \neq 0$.) Define $\mathbf{u}_{n-1}^{(A)}=\zeta_{n-1}^{(A)} \mathbf{v}_{A}$. Meanwhile, $\zeta_{n-1}^{(B)}$ is set to be a fixed value, and the mapping $\mathbf{u}_{n-1}^{(B)}$ is set to zero. It follows that

$$
\mathbf{x}_{n}=\mathbf{D}^{n} \mathbf{x}_{0}+\zeta_{n-1}^{(A)} \mathbf{E}_{A} \mathbf{v}_{A}
$$


Since one can uniquely identify $\mathbf{x}_{0}$ from the observation $\left\{\mathbf{C}_{B} \mathbf{x}_{0}, \mathbf{C}_{B} \mathbf{x}_{1}, \ldots\right.$, $\left.\mathbf{C}_{B} \mathbf{x}_{n-1}\right\}$, it follows from (5.12) that $\zeta_{n-1}^{(A)} \mathbf{C}_{B} \mathbf{E}_{A} \mathbf{v}_{A}$, and hence $\zeta_{n-1}^{(A)}$, is a function of $\left\{\mathbf{C}_{B} \mathbf{x}_{0}, \mathbf{C}_{B} \mathbf{x}_{1}, \ldots, \mathbf{C}_{B} \mathbf{x}_{n}\right\}$. So one can define $Q_{n}^{(B)}$ taking $\left\{\mathbf{C}_{B} \mathbf{x}_{0}, \mathbf{C}_{B} \mathbf{x}_{1}, \ldots, \mathbf{C}_{B} \mathbf{x}_{n}\right\}$ as arguments so that

$$
\psi_{n}^{(B)}=\zeta_{n-1}^{(A)}
$$

Define $\psi_{n}^{(A)}$ to be zero. This completes the description of a bit-sending cycle. At the end of this cycle Bob has knowledge of the bit sent by Alice, and both agents can proceed down one level in the protocol tree that defines $\Pi$. The rest of the bitsending sequence can be transcribed similarly. Note that two bits of communication are needed for every bit sent in $\Pi$ : one bit from one of agents to the dynamical system and one bit from the dynamical system to the other agent.

Depending on the values of $\alpha$ and $\beta$, determination of $f(\alpha, \beta)$ may take $D(f)$ or fewer cycles. But by definition, the second phase always completes at time $n+D(f)-1$ with the understanding that once the computation of $f(\alpha, \beta)$ is completed, no further communication bits are exchanged in this phase. So at the end of the second phase and after at most $2 D(f)$ bits have been exchanged, both agents have knowledge of $f(\alpha, \beta)$. It follows from (5.12) that the number of possible values of $\mathbf{x}_{n}$ is at most $2\left|\mathcal{X}_{0}\right|$; thus in general the number of possible values of $\mathbf{x}_{n+D(f)-1}$ is bounded from above by

$$
2^{D(f)}\left|\mathcal{X}_{0}\right|
$$

Since at the end of the second phase both agents have knowledge of $\mathbf{x}_{0}$ and all the controls used before time $n+D(f)-1$, the value of $\mathbf{x}_{n+D(f)-1}$ is known to both agents.

The final phase starts with the computation of $\zeta_{n+D(f)-1}^{(A)}$ and $\zeta_{n+D(f)-1}^{(B)}$ and ends at time $2 n+D(f)-1$. Let $\mathbf{x}^{(s)}$ denote $\mathbf{D}^{n} \mathbf{x}_{n+D(f)-1}$. Note that both agents have knowledge of $\mathbf{x}^{(s)}$ at the end of the second phase. By the definition of $\mathcal{B}_{A}$ and $\mathcal{B}_{B}$, there exist a vector sequence in $\mathbb{R}^{m_{A}},\left\{\mathbf{z}_{A}(1), \ldots, \mathbf{z}_{A}(n)\right\}$, and a vector sequence in $\mathbb{R}^{m_{B}},\left\{\mathbf{z}_{B}(1), \ldots, \mathbf{z}_{B}(n)\right\}$, satisfying

$$
\begin{aligned}
& P_{A} f(\alpha, \beta)=P_{A} \mathbf{x}^{(s)}+\mathbf{D}^{n-1} \mathbf{E}_{A} \mathbf{z}_{A}(1)+\mathbf{D}^{n-2} \mathbf{E}_{A} \mathbf{z}_{A}(2)+\cdots+\mathbf{E}_{A} \mathbf{z}_{A}(n), \\
& P_{B} f(\alpha, \beta)=P_{B} \mathbf{x}^{(s)}+\mathbf{D}^{n-1} \mathbf{E}_{B} \mathbf{z}_{B}(1)+\mathbf{D}^{n-2} \mathbf{E}_{B} \mathbf{z}_{B}(2)+\cdots+\mathbf{E}_{B} \mathbf{z}_{B}(n) .
\end{aligned}
$$

Hence,

$$
\begin{aligned}
& f(\alpha, \beta)=P_{A} f(\alpha, \beta)+P_{B} f(\alpha, \beta) \\
& =P_{A} \mathbf{x}^{(s)}+P_{B} \mathbf{x}^{(s)}+\mathbf{D}^{n-1}\left(\mathbf{E}_{A} \mathbf{z}_{A}(1)+\mathbf{E}_{B} \mathbf{z}_{B}(1)\right)+\cdots+\mathbf{E}_{A} \mathbf{z}_{A}(n)+\mathbf{E}_{B} \mathbf{z}_{B}(n) \\
& =\mathbf{D}^{n} \mathbf{x}_{n+D(f)-1}+\mathbf{D}^{n-1}\left(\mathbf{E}_{A} \mathbf{z}_{A}(1)+\mathbf{E}_{B} \mathbf{z}_{B}(1)\right)+\cdots+\mathbf{E}_{A} \mathbf{z}_{A}(n)+\mathbf{E}_{B} \mathbf{z}_{B}(n) .
\end{aligned}
$$

It follows that if one defines

$$
\begin{aligned}
& \mathbf{u}_{n+D(f)-1}^{(A)}=\mathbf{z}_{A}(1), \mathbf{u}_{n+D(f)}^{(A)}=\mathbf{z}_{A}(2), \ldots, \mathbf{u}_{n+D(f)+n-2}^{(A)}=\mathbf{z}_{A}(n), \\
& \mathbf{u}_{n+D(f)-1}^{(B)}=\mathbf{z}_{B}(1), \mathbf{u}_{n+D(f)}^{(B)}=\mathbf{z}_{B}(2), \ldots, \mathbf{u}_{n+D(f)+n-2}^{(B)}=\mathbf{z}_{B}(n),
\end{aligned}
$$


then system (4.1) is assured of reaching $f(\alpha, \beta)$ at time $2 n+D(f)-1$. The remaining task is to estimate the number of bits needed to code the control sequences. Since there are $\left|\mathcal{F}_{R}\right|$ possible values for $f(\alpha, \beta)$, it takes at most

$$
D(f)+\left\lceil\log _{2}\left|\mathcal{X}_{0}\right|\right\rceil+\left\lceil\log _{2}\left|\mathcal{F}_{R}\right|\right\rceil
$$

bits for Alice to uniquely identify the control sequence defined in (5.18). Similarly, at most $D(f)+\left\lceil\log _{2}\left|\mathcal{X}_{0}\right|\right\rceil+\left\lceil\log _{2}\left|\mathcal{F}_{R}\right|\right\rceil$ bits are needed for Bob to identify the control sequences in (5.19). So by prestoring all the possible control sequences of the form (5.18) and (5.19) at the dynamical system, and by using $\zeta_{n+D(f)-1}^{(A)}$ and $\zeta_{n+D(f)-1}^{(B)}$ to signal the control sequences, the system will reach $f(\alpha, \beta)$ at time $2 n+D(f)-1$. No further control code-words are needed from any of the agents afterwards. Counting all the bits in all three phases, one can obtain the upper bound in (5.8).

Remark. The conditions $\mathbf{C}_{B} \mathbf{E}_{A} \neq 0$ and $\mathbf{C}_{A} \mathbf{E}_{B} \neq 0$ ensure that control from one agent is immediately detectable by the other agent. Since $\left(\mathbf{D}, \mathbf{C}_{A}\right)$ and $\left(\mathbf{D}, \mathbf{C}_{B}\right)$ are observable, these conditions are not absolutely essential for Theorem 2. Nevertheless, it significantly simplifies the arguments.

TheOREm 3. Consider a distributed control system $(\boldsymbol{\Sigma}, f)$ with a Type B target function whose values are cells in lattice $\Lambda$. Suppose that the triple $\left(\mathbf{D}, \mathbf{E}_{A}, \mathbf{E}_{B}\right)$ is jointly controllable with a base $\mathcal{B}_{A} \cup \mathcal{B}_{B}, \mathbf{D}$ is invertible, the ordered pairs $\left(\mathbf{D}, \mathbf{C}_{A}\right)$ and $\left(\mathbf{D}, \mathbf{C}_{B}\right)$ are both observable, $\mathbf{C}_{B} \mathbf{E}_{A} \neq 0, \mathbf{C}_{A} \mathbf{E}_{B} \neq 0$, and the initial set $\mathcal{X}_{0}$ is bounded; then

$$
Y(\boldsymbol{\Sigma}, f) \leq 4 D(f)+4\left\lceil\log _{2}\left(O\left(\mathbf{D}^{2 n+D(f)-1} \mathcal{X}_{0}, \Lambda\right)\right)\right\rceil+2\left\lceil\log _{2}\left|\mathcal{F}_{R}\right|\right\rceil .
$$

Proof. Let $\Pi$ be a protocol which achieves the communication complexity $D(f)$ for the computation of $f$ according to the traditional model. The protocol for the distributed control system is divided into three phases as in the proof of Theorem 2.

The first phase starts from time 0 and ends when the quantized code-word of the observed state, $\mathbf{x}_{n-1}$, is communicated to the agents. In this phase all controls are set to zero. From the observability assumption, $\mathbf{x}_{0}$ can be determined from $\left\{\mathbf{C}_{A} \mathbf{x}_{0}, \mathbf{C}_{A} \mathbf{x}_{1}, \ldots, \mathbf{C}_{A} \mathbf{x}_{n-1}\right\}$ or from $\left\{\mathbf{C}_{B} \mathbf{x}_{0}, \mathbf{C}_{B} \mathbf{x}_{1}, \ldots, \mathbf{C}_{B} \mathbf{x}_{n-1}\right\}$ as shown in the proof of Theorem 2. The value $\mathbf{D}^{n+D(f)-1} \mathbf{x}_{0}$ is then quantized by mapping it to the cell in the lattice $\mathbf{D}^{-n} \Lambda$ to which it belongs. The values of the quantization functions $Q_{n-1}^{(A)}$ and $Q_{n-1}^{(B)}$ are the cell indices, the total number of which is bounded above by

$$
O\left(\mathbf{D}^{n+D(f)-1} \mathcal{X}_{0}, \mathbf{D}^{-n} \Lambda\right)=O\left(\mathbf{D}^{2 n+D(f)-1} \mathcal{X}_{0}, \Lambda\right) .
$$

Hence, the number of bits communicated in this phase is

$$
2\left\lceil\log _{2}\left(O\left(\mathbf{D}^{2 n+D(f)-1} \mathcal{X}_{0}, \Lambda\right)\right)\right\rceil .
$$

The second phase starts with the computation of $\zeta_{n-1}^{(A)}$ and $\zeta_{n-1}^{(B)}$ and ends at the delivery of the code-words $\psi_{n+D(f)-1}^{(A)}$ and $\psi_{n+D(f)-1}^{(B)}$ to the agents. In this phase, one transcribes $\Pi$ to the distributed control protocol. The approach is identical to that in the proof of Theorem 2. Note that a key point of this part of the proof is to show that the control code-words are functions of the observation sequences of the form $\left\{\mathbf{C}_{A} \mathbf{x}_{0}, \mathbf{C}_{A} \mathbf{x}_{1}, \ldots, \mathbf{C}_{A} \mathbf{x}_{n-1}\right\}$ or $\left\{\mathbf{C}_{B} \mathbf{x}_{0}, \mathbf{C}_{B} \mathbf{x}_{1}, \ldots, \mathbf{C}_{B} \mathbf{x}_{n-1}\right\}$. This issue does not depend on whether the initial set is finite or not. In this phase, at most $2 D(f)$ bits are communicated. 
The third and final phase starts with the computation of the code-words $\zeta_{n+D(f)-1}^{(A)}$ and $\zeta_{n+D(f)-1}^{(B)}$ and ends at time $2 n+D(f)-1$. Note that

$$
\mathbf{x}_{n+D(f)-1}=\mathbf{D}^{n+D(f)-1} \mathbf{x}_{0}+\mathbf{w}(\alpha, \beta),
$$

where $\mathbf{w}(\alpha, \beta)$ represents the total control effect from Alice and Bob up to $t=$ $n+D(f)-2$ and has at most $2^{D(f)}$ different values. Moreover, since both agents have knowledge of these control values, $\mathbf{w}(\alpha, \beta)$, which is independent of $\mathbf{x}_{0}$, can be determined by both agents.

Let $\left\langle\mathbf{D}^{n+D(f)-1} \mathbf{x}_{0}\right\rangle$ represent the center of the cell in the lattice $\mathbf{D}^{-n} \Lambda$ to which $\mathbf{D}^{n+D(f)-1} \mathbf{x}_{0}$ belongs. The value of $\left\langle\mathbf{D}^{n+D(f)-1} \mathbf{x}_{0}\right\rangle$ is known to both agents. Let $\mathbf{x}^{(f)}$ denote the center of the cell $f(\alpha, \beta)$. The code-words $\zeta_{n+D(f)-1}^{(A)}$ and $\zeta_{n+D(f)-1}^{(B)}$ are designed to trigger control sequences that jointly steer $\left\langle\mathbf{D}^{n+D(f)-1} \mathbf{x}_{0}\right\rangle+\mathbf{w}(\alpha, \beta)$ to $\mathbf{x}^{(f)}$ in $n$ steps. Thus we have

$$
\begin{aligned}
\mathbf{x}^{(f)}= & \mathbf{D}^{n}\left\langle\mathbf{D}^{n+D(f)-1} \mathbf{x}_{0}\right\rangle+\mathbf{D}^{n} \mathbf{w}(\alpha, \beta)+\mathbf{D}^{n-1}\left(\mathbf{E}_{A} \mathbf{u}_{n+D(f)-1}^{(A)}+\mathbf{E}_{B} \mathbf{u}_{n+D(f)-1}^{(B)}\right) \\
& +\mathbf{D}^{n-2}\left(\mathbf{E}_{A} \mathbf{u}_{n+D(f)}^{(A)}+\mathbf{E}_{B} \mathbf{u}_{n+D(f)}^{(B)}\right)+\cdots \\
& +\mathbf{E}_{A} \mathbf{u}_{n+D(f)+n-2}^{(A)}+\mathbf{E}_{B} \mathbf{u}_{n+D(f)+n-2}^{(B)} \\
\equiv & \mathbf{D}^{n}\left\langle\mathbf{D}^{n+D(f)-1} \mathbf{x}_{0}\right\rangle+\mathbf{D}^{n} \mathbf{w}(\alpha, \beta)+\mathbf{q}
\end{aligned}
$$

for some control sequences $\left\{\mathbf{u}_{t}^{(A)}\right\}$ and $\left\{\mathbf{u}_{t}^{(B)}\right\}$. Details of this construction are similar to the approach explained in the proof of Theorem 2 and are not repeated here. Under these control sequences, the trajectory is steered to the state

$$
\begin{aligned}
\mathbf{x}_{2 n+D(f)-1} & =\mathbf{D}^{n} \mathbf{x}_{n+D(f)-1}+\mathbf{q} \\
& =\mathbf{D}^{n} \mathbf{x}_{n+D(f)-1}+\mathbf{x}^{(f)}-\mathbf{D}^{n}\left\langle\mathbf{D}^{n+D(f)-1} \mathbf{x}_{0}\right\rangle-\mathbf{D}^{n} \mathbf{w}(\alpha, \beta) \\
& =\mathbf{x}^{(f)}+\mathbf{D}^{2 n+D(f)-1} \mathbf{x}_{0}-\mathbf{D}^{n}\left\langle\mathbf{D}^{n+D(f)-1} \mathbf{x}_{0}\right\rangle .
\end{aligned}
$$

Let $\Lambda$ be generated by the base $\mathbf{Z}=\left(\mathbf{z}_{1}, \ldots, \mathbf{z}_{n}\right)$. By definition, a cell center in the lattice $\mathbf{D}^{-n} \Lambda$ satisfies

$$
\mathbf{D}^{n+D(f)-1} \mathbf{x}_{0}-\left\langle\mathbf{D}^{n+D(f)-1} \mathbf{x}_{0}\right\rangle=\sum_{i=1}^{n} \alpha_{i} \mathbf{D}^{-n} \mathbf{z}_{i}
$$

with $-1 / 2 \leq \alpha_{i}<1 / 2$ for $i=1, \ldots, n$. Hence,

$$
\begin{aligned}
\mathbf{x}_{2 n+D(f)-1}-\mathbf{x}^{(f)} & =\mathbf{D}^{n}\left(\mathbf{D}^{n+D(f)-1} \mathbf{x}_{0}-\left\langle\mathbf{D}^{n+D(f)-1} \mathbf{x}_{0}\right\rangle\right) \\
& =\mathbf{D}^{n} \sum_{i=1}^{n} \alpha_{i} \mathbf{D}^{-n} \mathbf{z}_{i}=\sum_{i=1}^{n} \alpha_{i} \mathbf{z}_{i} .
\end{aligned}
$$

Therefore, the state $\mathbf{x}_{2 n+D(f)-1}$ is contained in the cell $f(\alpha, \beta)$ in the lattice $\Lambda$. The number of code-words needed to represent the control sequence for either Alice or Bob is bounded by

$$
\left|\mathcal{F}_{R}\right| 2^{D(f)} O\left(\mathbf{D}^{n+D(f)-1} \mathcal{X}_{0}, \mathbf{D}^{-n} \Lambda\right)=\left|\mathcal{F}_{R}\right| 2^{D(f)} O\left(\mathbf{D}^{2 n+D(f)-1} \mathcal{X}_{0}, \Lambda\right) .
$$


Hence, at most

$$
2 D(f)+2\left\lceil\log _{2}\left(O\left(\mathbf{D}^{2 n+D(f)-1} \mathcal{X}_{0}, \Lambda\right)\right)\right\rceil+2\left\lceil\log _{2}\left|\mathcal{F}_{R}\right|\right\rceil
$$

bits are communicated at phase 3 . The theorem then follows.

Theorems 2 and 3 show that there are cases for which the control communication complexity is finite and hence well defined. The upper bounds, however, are not tight. It should also be pointed out that the term $4\left\lceil\log _{2}\left(O\left(\mathbf{D}^{2 n+D(f)-1} \mathcal{X}_{0}, \Lambda\right)\right)\right\rceil$ in the upper bound can be improved if Alice and Bob can send some of their bits in parallel rather than in sequence.

The quantization functions of the observations are allowed to depend on the full value of past observations. If the initial state set is finite, it is possible to develop results similarly using quantization functions that are based on quantized values of past observations.

\section{More examples and general properties.}

Example 1. Consider the one-dimensional system

$$
\left(E_{1}\right)\left\{\begin{array}{l}
x_{t+1}=a x_{t}+u_{t}^{(A)}+u_{t}^{(B)}, \quad x_{0} \in \mathcal{X}_{0} \subseteq \mathbb{R}, \\
y_{t}=C_{A} x_{t}=C_{B} x_{t}=x_{t},
\end{array}\right.
$$

where $\mathcal{X}_{0}=\left\{\delta, \ldots, 2^{J} \delta\right\} \subseteq \Lambda=\{i \delta: i \in \mathbb{Z}\}$. Let $f$ be a function from $\mathcal{A} \times \mathcal{B}$ to $\mathcal{X}_{0}$ with a communication complexity $D(f)$. Theorem 1 implies the following inequality:

$$
2 D(f) \leq Y(\boldsymbol{\Sigma}, f) .
$$

To obtain a sharper upper bound, let $\Pi$ be a protocol that achieves the communication complexity $D(f)$. Construct a three-phase protocol similar to Theorem 2. A major difference is that at the end of phase 1 , the state information is sent only to Alice and the control in phase 3 is triggered only by Alice. Moreover, in the second phase, the control values used to indicate the bit sequence in the second phase are chosen so that $u_{t}^{(*)} \in\{\delta, 0\}$. It follows that the state $\mathbf{x}_{n+D(f)-1}$ is of the form

$$
\left[a^{D(f)} i_{0}+\left(c_{1} a^{D(f)-1}+c_{2} a^{D(f)-2}+\cdots+c_{D(f)}\right)\right] \delta,
$$

with $c_{i} \in\{0,1\}$ and $x_{0}=i_{0} \delta$. If $a$ is a transcendental number, such as $\pi$, then the set of all such elements has exactly

$$
2^{J+D(f)}
$$

distinct elements. However, the order of such a set might be reduced if $a$ is an algebraic number. For example, if $a=1$, then all elements represented by (6.3) are of the form $i \delta$ for some integer $i$, with $1 \leq i \leq 2^{J}+D(f)$, and it takes at most

$$
\left\lceil\log _{2}\left(2^{J}+D(f)\right)\right\rceil+\left\lceil\log _{2}\left|\mathcal{F}_{R}\right|\right\rceil
$$

bits to code the control sequences that steer the system to the target set. In this case, the control communication complexity is subject to a sharper upper bound:

$$
2 D(f)+\left\lceil\log _{2}\left|\mathcal{X}_{0}\right|\right\rceil+\left\lceil\log _{2}\left(D(f)+\left|\mathcal{X}_{0}\right|\right)\right\rceil+\left\lceil\log _{2}\left|\mathcal{F}_{R}\right|\right\rceil .
$$

Example 2. The upper bound of $Y(\boldsymbol{\Sigma}, f)$ in (5.8) contains the term $\left\lceil\log _{2}\left|\mathcal{X}_{0}\right|\right\rceil$. A natural question is whether the lower bound of $Y(\boldsymbol{\Sigma}, f)$ should also contain such a 
term. The following example shows that this is not true in general. In fact, the lower bound is tight for some systems. Consider

$$
\left(E_{2}\right)\left\{\begin{array}{l}
\mathbf{x}_{t+1}=\left(\begin{array}{ll}
0 & 1 \\
0 & 0
\end{array}\right) \mathbf{x}_{t}+\left(\begin{array}{c}
0 \\
1
\end{array}\right)\left(u_{t}^{(A)}+u_{t}^{(B)}\right), \quad \mathbf{x}_{0} \in \mathcal{X}_{0}, \\
y_{t}=\mathbf{C}_{A} \mathbf{x}_{t}=\mathbf{C}_{B} \mathbf{x}_{t}=\left(\begin{array}{ll}
0 & 1)^{T} \mathbf{x}_{t}=\mathbf{x}_{t}[1] .
\end{array}\right.
\end{array}\right.
$$

Let $\mathcal{A}$ and $\mathcal{B}$ be arbitrary finite sets and let $f$ be a Boolean function defined on $\mathcal{A} \times \mathcal{B}$. Recall that $C(f)$ denotes the original communication complexity defined by Yao [24]. Assume that $C(f)$ is related to $D(f)$ by

$$
D(f)=C(f)+1 .
$$

(One can take the example shown in section 3.) Let $\Pi$ be a protocol that achieves the communication complexity $D(f)$, with the last bit conveying the value of $f(\alpha, \beta)$ sent, without loss of generality, by Alice. One can extend $f$ to a function from $\mathcal{A} \times \mathcal{B}$ to $\mathbb{R}^{2}$ by mapping 0 to $(0,0)^{T}$ and 1 to $(1,0)^{T}$. Define a control system using this extended function. Since

$$
\left(\begin{array}{ll}
0 & 1 \\
0 & 0
\end{array}\right)^{2} \mathbf{x}_{0}=\mathbf{0}
$$

by setting

$$
u_{0}^{(A)}=u_{0}^{(B)}=u_{1}^{(A)}=u_{1}^{(B)}=0
$$

one can guarantee that

$$
\mathbf{x}_{2}=\mathbf{0}
$$

Construct a distributed control protocol $\Omega$ by extending the sequence in (6.10). For $t \geq 2$, set the control function by adapting the bit patterns of $\Pi$ to $\Omega$ according to the approach stated in the proof of Theorem 2. In particular, $u_{t}^{(A)}$ is equal to the bit value sent by Alice, and $u_{t}^{(B)}$ is equal to the bit value sent by Bob, at time $t$. At other times, set the control to 0 . Then it follows that

$$
\mathbf{x}_{D(f)+3}=\left(\begin{array}{ll}
0 & 1 \\
0 & 0
\end{array}\right)\left(\begin{array}{l}
0 \\
1
\end{array}\right) \mathbf{u}_{D(f)+1}^{(A)}=f(\alpha, \beta) .
$$

So in effect the final bit in the communication phase is also used to drive the system to the target at time $D(f)+3$. In the protocol $\Omega$, the system does not send to the agents any bits unrelated to $\Pi$. Hence,

$$
Y(\boldsymbol{\Sigma}, f)=2 D(f) .
$$

This proves the following.

Proposition 1. For the dynamical system defined in (6.7), the lower bound in (5.1) is achieved by a distributed control protocol.

Although the lower bound in (5.8) is tight for some systems, it can be improved in general to include the effect of the initial set size and system dynamics. Exploration in this direction is beyond the scope of the current paper. However, influence of 
system dynamics on the control communication complexity can be easily observed in the upper bound. For example, if the set $\mathcal{D}=\bigcup_{i \geq 0} \mathbf{D}^{i} \mathcal{X}_{0}$ is bounded, then, for all $i \geq 0$,

$$
O\left(\mathbf{D}^{i} \mathcal{X}_{0}, \Lambda\right) \leq M
$$

for some $M$, and the upper bound in (5.21) can be reformulated as

$$
Y(\boldsymbol{\Sigma}, f) \leq 4 D(f)+4\left\lceil\log _{2} M\right\rceil+2\left\lceil\log _{2}\left|\mathcal{F}_{R}\right|\right\rceil .
$$

Example 3. Consider the control communication complexity of a simple two-way communication system that allows Alice to send one bit to Bob and Bob to send one bit to Alice. One can implement this via the so-called equality function EQ (see [13]). (For multiple bit communication, one can consider a direct sum of EQ.) table:

If $\mathcal{A}=\mathcal{B}=\{0,1\}$, then the EQ function can be represented by the following

\begin{tabular}{|c|c|c|}
\hline$\beta \backslash \alpha$ & 0 & 1 \\
\hline 0 & 1 & 0 \\
\hline 1 & 0 & 1 \\
\hline
\end{tabular}

If Alice and Bob can determine the value $E Q(\alpha, \beta)$, where $\alpha$ and $\beta$ are the choice of Alice and Bob, respectively, then Alice can retrieve the bit sent by Bob by using the operation

$$
\mathrm{EQ}(\alpha, \beta) \oplus \alpha \oplus 1,
$$

where $\oplus$ denotes the mod 2 addition. Similarly, Bob can also retrieve the bit sent by Alice by the operation

$$
\operatorname{EQ}(\alpha, \beta) \oplus \beta \oplus 1 \text {. }
$$

It is well known that the communication complexity of EQ is 2. One can extend this problem to a control dynamical system. To do so, extend the EQ function to a function from $\mathcal{A} \times \mathcal{B}$ to $\mathbb{R}^{2}$ by identifying 0 with $[0,1]^{T}$ and 1 with $[1,0]^{T}$. Label this function CEQ. Now consider the system

$$
\left(E_{3}\right)\left\{\begin{array}{l}
\mathbf{x}_{t+1}=\left(\begin{array}{ll}
0 & 1 \\
1 & 0
\end{array}\right) \mathbf{x}_{t}+\left(\begin{array}{c}
-1 \\
1
\end{array}\right)\left(u_{t}^{(A)}+u_{t}^{(B)}\right), \quad \mathbf{x}_{0} \in \mathcal{X}_{0}=\left\{\left[\begin{array}{l}
1 \\
0
\end{array}\right],\left[\begin{array}{l}
0 \\
1
\end{array}\right]\right\}, \\
y_{t}=\mathbf{C}_{A} \mathbf{x}_{t}=\mathbf{C}_{B} \mathbf{x}_{t}=(10)^{T} \mathbf{x}_{t}=\mathbf{x}_{t}[1] .
\end{array}\right.
$$

Proposition 2. The control communication complexity for the distributed control system defined by (6.18) and the CEQ function is 6.

Proof. One can achieve the objective with six communication bits. To show this, define a quantization function,

$$
P(s)= \begin{cases}0 & \text { if } s \leq 0 \\ 1 & \text { if } s>0\end{cases}
$$

Define the observation coding at time 0 by

$$
\psi_{0}^{(A)}=\psi_{0}^{(B)}=P\left(y_{0}\right)
$$


Thus, 0 indicates that the system starts at $[0,1]^{T}$, and 1 indicates that the system starts at $[1,0]^{T}$. Alice then uses $\zeta_{0}^{(A)}$ to indicate her choice to Bob by using the following equations:

$$
\begin{aligned}
& \zeta_{0}^{(A)}=\alpha, \quad \zeta_{0}^{(B)}=u_{0}^{(B)}=0, \\
& u_{0}^{(A)}= \begin{cases}1 & \text { if } \psi_{0}^{(A)}=0 \text { and } \zeta_{0}^{(A)}=1, \\
0 & \text { otherwise, }\end{cases} \\
& \psi_{1}^{(A)}=\psi_{1}^{(B)}=\zeta_{1}^{(A)}=\zeta_{1}^{(B)}=u_{1}^{(B)}=0, \\
& u_{1}^{(A)}= \begin{cases}1 & \text { if } \psi_{0}^{(A)}=1 \text { and } \zeta_{0}^{(A)}=1, \\
0 & \text { otherwise, }\end{cases} \\
& \psi_{2}^{(A)}=0, \quad \psi_{2}^{(B)}=P\left(y_{2}\right) .
\end{aligned}
$$

Two bits are communicated so far, one for $\zeta_{0}^{(A)}$ and one for $\psi_{2}^{(B)}$. One can check

$$
\begin{aligned}
& \left(\psi_{0}^{(B)}=0\right) \wedge(\alpha=0) \Rightarrow \psi_{2}^{(B)}=0, \quad\left(\psi_{0}^{(B)}=1\right) \wedge(\alpha=1) \Rightarrow \psi_{2}^{(B)}=0, \\
& \left(\psi_{0}^{(B)}=1\right) \wedge(\alpha=0) \Rightarrow \psi_{2}^{(B)}=1, \quad\left(\psi_{0}^{(B)}=0\right) \wedge(\alpha=1) \Rightarrow \psi_{2}^{(B)}=1 .
\end{aligned}
$$

Hence, Bob can deduce the value of $\alpha$ from $\psi_{2}^{(B)}$ and $\psi_{0}^{(B)}$. This completes the task of sending information from Alice to Bob. Bob sends his choice to Alice via $\zeta_{2}^{(B)}$ in a similar way. Since the system always remains in $\mathcal{X}_{0}$, it reaches $f(\alpha, \beta)$ at either $t=4$ or $t=5$. The exact time can be deduced by both agents based on information of the initial state and the choices of $\alpha$ and $\beta$. Hence, the upper bound for the communication complexity is six.

To show that the lower bound of the communication control complexity is six, first note that for this target function, Alice must send at least one bit to Bob via the system and Bob must send one bit to Alice via the system in relation to the computation of the EQ function. So, at least four bits are required, of which two are control code bits and two are observation code bits. Call these the basic bits.

If each agent receives at least two observation bits, then at least six bits must be communicated. Suppose one of the agents, say Alice, receives only one observation code bit; that bit must then be a basic bit. The value of that basic bit is arbitrary depending on Bob's choice of $\beta$. Hence, Alice does not have knowledge of $\mathbf{x}_{0}$. In order for Alice to have knowledge of $\tau$, the time the target state is reached, it must be independent of $\mathbf{x}_{0}$. On the other hand, one can show from direct examination of (6.18) that if the controls $u_{t}^{(A)}$ and $u_{t}^{(B)}$ are independent of $\mathbf{x}_{0}$, then $\mathbf{x}_{\tau}$ assumes a different value for a different initial state. Therefore, $u_{t}^{(B)}$ must be dependent on $\mathbf{x}_{0}$ and Bob must have received at least one additional observation bit other than the basic bit which enables him to determine $\mathbf{x}_{0}$. Moreover, the control that drives the system to $f(\alpha, \beta)$ cannot be shared with the basic control bit, since a basic control bit must have two possible states as outcome in order to code the information from an agent. Thus the lower bound is six.

Example 4. For the case where $\mathcal{F}_{R}$ is a singleton, say containing just the origin, the traditional communication complexity is zero and the control communication complexity can be viewed as a measure of the complexity of a stabilizing feedback algorithm. Under this interpretation, the control communication complexity is related 
to the complexity introduced by Fagnani and Zampieri [10]. However, there is at least one major difference. In [10], computation complexity of the controller state is also included, while here only communication complexity is considered.

7. Conclusion. In this paper, the concept of communication complexity is extended to a two-agent distributed control system. This new perspective connects communication complexity, a classical idea from distributed computing theory, with ideas from control systems under communication constraints. The concept of control communication complexity is defined, and some of its fundamental properties are presented. It is possible to extend the ideas presented here in several directions. One direction is to define a control communication complexity for a multiagent distributed control system. This is an important class of problems given the emergence of peer-to-peer networks such as the Internet and wireless ad hoc networks. An early investigation on multiagent, low data-rate, distributed controllers can be found in [23]. In many control and optimization problems, the target states are defined only implicitly; how to define the control communication complexity for such systems is another interesting direction for extension.

Acknowledgments. The author would like to thank the reviewers, the associate editor, and Professor John Baillieul for their valuable comments.

\section{REFERENCES}

[1] J. Baillieul, Feedback designs in information-based control, Stochastic Theory and Control, Lecture Notes in Control and Inform. Sci. 280, Springer, Berlin, 2002, pp. 35-57.

[2] A. Berman and R. J. Plemmons, Nonnegative Matrices in the Mathematical Sciences, Academic Press, New York, 1994.

[3] R. W. Brockett and D. Liberzon, Quantized feedback stabilization of linear systems, IEEE Trans. Automat. Control, 45 (2000), pp. 1279-1289.

[4] J. Conway and N. J. A. Sloane, Sphere Packings, Lattices and Groups, Springer, Berlin, 1999.

[5] C. De Persis And A. Isidori, Stabilizability by state feedback implies stabilizability by encoded state feedback, Systems Control Lett., 53 (2004), pp. 249-258.

[6] D. F. Delchamps, Stabilizing a linear system with quantized state feedback, IEEE Trans. Automat. Control, 35 (1990), pp. 916-924.

[7] N. Elia And S. K. Mitter, Stabilization of linear systems with limited information, IEEE Trans. Automat. Control, 49 (2001), pp. 1384-1400.

[8] F. Fagnani, Chaotic quantized feedback stabilizers: The scalar case, Commun. Inf. Syst., 4 (2004), pp. 53-72.

[9] F. FAGnani And S. Zampieni, Stability analysis and synthesis for scalar linear systems with a quantized feedback, IEEE Trans. Automat. Control, 48 (2003), pp. 1569-1584.

[10] F. FAGnani And S. Zampieri, Quantized stabilization of linear systems: Complexity versus performance, IEEE Trans. Automat. Control, 49 (2004), pp. 1534-1548.

[11] Y. C. Ho, Team decision theory and information structures, Proc. IEEE, 68 (1980), pp. 644654.

[12] N. Ishit And B. A. Francis, Limited Data Rate in Control Systems with Networks, Lecture Notes in Control and Inform. Sci. 275, Springer, Berlin, 2002.

[13] E. Kushilevitz and N. Nisan, Communication Complexity, Cambridge University Press, Cambridge, UK, 1997.

[14] W. LeOntiEF, Lags and the stability of dynamic systems, Econometrica, 29 (1961), pp. 659-669.

[15] K. Li AND J. BAillieul, Robust quantization for digital finite communication bandwidth (DFCB) control, IEEE Trans. Automat. Control, 49 (2004), pp. 1573-1584.

[16] D. G. Luenberger and A. Arbel, Singular dynamic Leontief systems, Econometrica, 45 (1977), pp. 991-995.

[17] G. N. NAIR And R. J. Evans, Stabilization with data-rate-limited feedback: Tightest attainable bounds, Systems Control Lett., 41 (2000), pp. 49-56.

[18] G. N. NAIR AND R. J. Evans, Exponential stabilisability of finite-dimensional linear systems with limited data rates, Automatica J. IFAC, 39 (2003), pp. 585-593. 
[19] S. Tatikonda And S. K. Mitter, Control under communication constraints, IEEE Trans. Automat. Control, 49 (2004), pp. 1056-1068.

[20] S. Tatikonda, A. Sahai, And S. Mitter, Control of LQG systems under communication constraints, in Proceedings of the 37th IEEE Conference on Decision and Control, Vol. 1, Tampa, FL, 1998, pp. 1165-1170.

[21] W. S. Wong AND R. W. BRockett, Systems with finite communication bandwidth constraints I: State estimation problems, IEEE Trans. Automat. Control, 42 (1997), pp. 12941298.

[22] W. S. Wong And R. W. Brockett, Systems with finite communication bandwidth constraints II: Stabilization with limited information feedback, IEEE Trans. Automat. Control, 44 (1999), pp. 1049-1053.

[23] W. S. Wong And C. W. Sung, Robust convergence of low data rate-distributed controllers, IEEE Trans. Automat. Control, 49 (2004), pp. 82-87.

[24] A. C.-C. YAO, Some complexity questions related to distributive computing, in Proceedings of the 11th Annual ACM Symposium on Theory of Computing (STOC), 1979, pp. 209-213. 